\title{
ESPECTROS ELETRÔNICOS DE ALGUNS COMPLEXOS DE GEOMETRIA OCTAÉDRICA DE Ni²+: UMA INTRODUÇÃO PRÁTICA À TEORIA DO CAMPO CRISTALINO NO CURSO DE GRADUAÇÃo
}

Yoshitaka Gushikem

Instituto de Quimica, Universidade Estadual de Campinas, CP 6154, 13084-971 Campinas - SP

Recebido em 26/11/03; aceito em 18/5/04; publicado na web em 9/8/04

\begin{abstract}
ELECTRONIC SPECTRA OF SOME $\mathrm{Ni}^{2+}$ OCTAHEDRAL COMPLEXES: A PRACTICAL INTRODUCTION TO THE CRYSTAL FIELD THEORY IN THE UNDERGRADUATE COURSE. The experiment introduces the undergraduate students to the crystal field theory. The electronic spectra of the octahedral complexes of $\left[\mathrm{Ni}(\mathrm{L})_{\mathrm{n}}\right]^{2+}\left(\mathrm{L}=\mathrm{H}_{2} \mathrm{O}\right.$ dmso, $\mathrm{NH}_{3}$ and en) obtained in the experiment are used to calculate $10 \mathrm{Dq}$ and $\mathrm{B}$ parameters. The experiment shows how the parameters can be calculated and correlated with the nature of the ligands and the field intensities produced.
\end{abstract}

Keywords: $\mathrm{Ni}(\mathrm{II})$ octahedral complexes; electronic spectra; crystal field parameters.

\section{INTRODUÇÃO}

Os complexos de metais de transição são fascinantes para os estudantes porque possuem uma variedade de cores e para um determinado metal central, esta mudança de coloração também pode ser observada, em função dos ligantes. Por exemplo, os compostos $\left[\mathrm{Co}\left(\mathrm{OH}_{2}\right)_{6}\right]^{2+}$ e $\mathrm{CoCl}_{4}^{2-}$ possuem o mesmo metal central mas apresentam cores diferentes, respectivamente rosada e azulada. Estes complexos absorvem a radiação eletromagnética de comprimento de onda $\lambda$ quando a luz natural (luz branca) incide sobre eles. As diferentes cores observadas são resultados das luzes refletidas cujas naturezas são diferentes para cada complexo. Os fundamentos básicos sobre esta questão os estudantes podem ler no livro texto de Atkins e Jones ${ }^{1}$.

A absorção da radiação eletromagnética ocorre quando o fóton incidente sobre um sistema promove uma transição entre os níveis de energia $\mathrm{E}_{i}$ e $\mathrm{E}_{j}$ cuja diferença é descrita pela equação $\Delta \mathrm{E}_{i j}=\mathrm{h} / \lambda$, onde h é a constante de Planck. Nos complexos de metais de transição, com configuração eletrônica $\mathrm{d}^{\mathrm{n}}(\mathrm{n}>1)$, as transições ocorrem entre níveis de energia resultantes da interação entre os elétrons d, sendo designadas de transições $\mathrm{d}-\mathrm{d}^{2}$.

Com o intuito de introduzir aos estudantes de graduação os fundamentos da teoria de campo cristalino, um experimento simples no qual os mesmos preparam e analisam os espectros de complexos hexacoordenados de $\mathrm{NiL}_{\mathrm{n}}^{2+}\left(\mathrm{L}=\mathrm{H}_{2} \mathrm{O}\right.$ dmso (dimetilsulfóxido), $\mathrm{NH}_{3}$ e en (etilenodiamina ou 1,2-diaminoetano) é proposto neste trabalho. A partir das transições eletrônicas devidamente atribuídas, os parâmetros $10 \mathrm{Dq}$ e de repulsão inter eletrônica B são calculados e correlacionados com a natureza dos ligantes. $\mathrm{O}$ experimento requer a utilização de espectrofotômetro UV-Visível, sensível na região compreendendo entre 300 a $800 \mathrm{~nm}$.

\section{PARTE EXPERIMENTAL}

Prepare inicialmente $20 \mathrm{~mL}$ de uma solução aquosa de $\mathrm{Ni}\left(\mathrm{NO}_{3}\right)_{2} \cdot 6 \mathrm{H}_{2} \mathrm{O} 0,1 \mathrm{~mol} \mathrm{~L}-1$. Pipete $5 \mathrm{ml}$ desta solução em dois erlenmeyers e coloque $5 \mathrm{~mL}$ de $\mathrm{NH}_{4} \mathrm{OH}$ concentrado no primeiro e no segundo, $5 \mathrm{~mL}$ de etilenodiamina pura. Prepare uma terceira solução dissolvendo diretamente o $\mathrm{Ni}\left(\mathrm{NO}_{3}\right)_{2} \cdot 6 \mathrm{H}_{2} \mathrm{O}$ sólido $(0,15 \mathrm{~g})$ em

*e-mail: gushikem@iqm.unicamp.br
$10 \mathrm{~mL}$ de dmso puro para que no final se tenha uma solução 0,05 mol L-1. Registre os espectros UV-visível para todas as amostras usando uma concentração de $0,05 \mathrm{~mol} \mathrm{~L}^{-1}$ em uma cubeta de quartzo, com $1 \mathrm{~cm}$ de caminho óptico.

$\mathrm{O}$ equipamento utilizado neste artigo foi o espectrofotômetro UV-Vis Shimadzu 1601PC com arranjo diodo entre 300 a $800 \mathrm{~nm}$. Os espectros obtidos são apresentados nas Figuras 1a-d.

\section{ANÁLISE DOS DADOS}

$\mathrm{O} \mathrm{Ni}^{2+}$ possui a configuração eletrônica $\mathrm{d}^{8}$ e possui, portanto, dois elétrons desemparelhados. Lembrando que cada elétron girando ao redor do núcleo gera um momento orbital, $l$, ocorre uma interação entre estes momentos individuais $l_{1}$ e $l_{2}$, referentes aos elétrons 1 e 2 (ver figura abaixo). O momento resultante possui magnitude expressa pela soma vetorial dos momentos individuais $|L|=\left|l_{i}\right|+\left|l_{j}\right|$.

$\begin{aligned} & l_{2}=1 \\ & l_{1}=2\end{aligned} \uparrow \uparrow L=3$

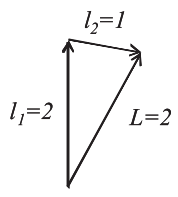

$l_{1}=2 \underset{\uparrow^{l_{2}=1}}{\uparrow_{L=1}}$

Soma vetorial de $l_{1} \mathrm{e}$ $l_{2}$ resulta nos momentos orbitais $L=1$, $L=2$ e $L=3$

Como os elétrons possuem individualmente um momento de spin $s$, a magnitude da resultante da interação é expressa por $|\mathrm{S}|=\left|s_{1}\right|$ $+\left|s_{2}\right|$. Pode ainda ocorrer uma interação denominada de spin-órbita $S+L$. Entretanto, a magnitude deste acoplamento, em comparação com os acoplamentos individuais $S$ e $L$ ser muito menor, não será considerada. Para um sistema que possui um ou mais elétrons na sua camada de valência, os estados espectroscópicos resultantes são representados por ${ }^{2 S+1} L$, onde $2 S+1$ é a multiplicidade do spin e $L$, o momento orbital resultante.

No íon gasoso (campo esférico), o íon $\mathrm{Ni}^{2+}$ apresenta, em decorrência das interações acima citadas e obedecendo-se o princípio de exclusão de Pauli, os seguintes estados de energia, denominados de termos de Russel-Saunders, são originados: ${ }^{3} \mathrm{~F},{ }^{3} \mathrm{P},{ }^{1} \mathrm{~S},{ }^{1} \mathrm{D}$ e ${ }^{1} \mathrm{G}$. Os alunos interessados em entender melhor como os elétrons interagem entre si em sistemas polieletrônicos, devem ler o texto de Herzberg ${ }^{3}$, sem dúvida muito didático e, portanto, o mais recomendado. 

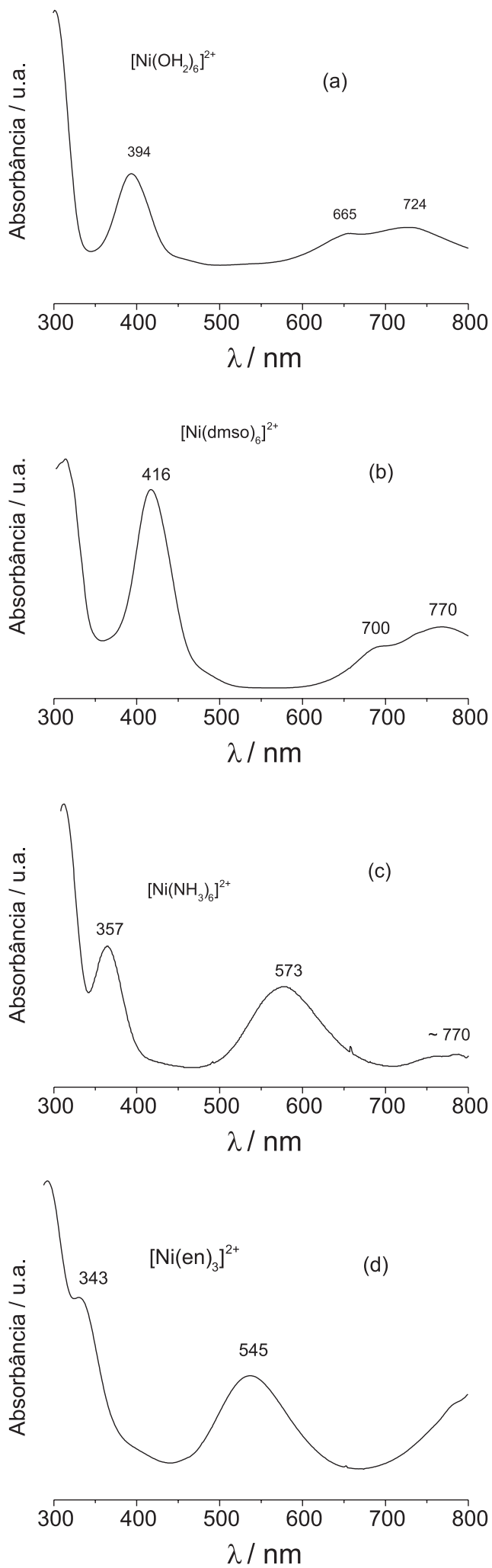

Figura 1. Espectros de absorção na região UV-visivel dos complexos de: (a) $\left[\mathrm{Ni}\left(\mathrm{H}_{2} \mathrm{O}\right)_{6}\right]^{2+}$, (b) $\left[\mathrm{Ni}(\mathrm{dmso})_{6}\right]^{2+},(\mathrm{c})\left[\mathrm{Ni}\left(\mathrm{NH}_{3}\right)_{6}\right]^{2+} e(d)\left[\mathrm{Ni}(\mathrm{en})_{3}\right]^{2+}$

Sob a ação do campo externo (Tabela 1), os termos de RusselSaunders desdobram-se e a ordem dos níveis de energia pode ser determinada através do diagrama de correlação. Neste ponto, os alunos devem ler o texto básico de Cotton $^{4}$ onde o autor demonstra detalhadamente como este diagrama pode ser construído, observando-se os princípios da mecânica quântica.
Tabela 1. Termos de Russel-Saunders para o íon gasoso e o desdobramento dos mesmos sob a ação de um campo $\mathrm{O}_{\mathrm{h}}$

\begin{tabular}{cl}
\hline Íon gasoso & $\begin{array}{l}\text { Estados resultantes sob a } \\
\text { ação de campo de grupo pontual } \mathrm{O}_{\mathrm{h}}\end{array}$ \\
\hline${ }^{3} \mathrm{~F}$ & ${ }^{3} \mathrm{~A}_{2 \mathrm{~g}},{ }^{3} \mathrm{~T}_{1 \mathrm{~g}},{ }^{3} \mathrm{~T}_{2 \mathrm{~g}}$ \\
${ }^{3} \mathrm{P}$ & ${ }^{3} \mathrm{~T}_{1 \mathrm{~g}}$ \\
${ }^{1} \mathrm{~S}$ & ${ }^{1} \mathrm{~A}_{1 \mathrm{~g}}$ \\
${ }^{1} \mathrm{D}$ & ${ }^{1} \mathrm{E}_{\mathrm{g}},{ }^{1} \mathrm{~T}_{2 \mathrm{~g}}$ \\
${ }^{1} \mathrm{G}$ & ${ }^{1} \mathrm{~A}_{1 \mathrm{~g}},{ }^{1} \mathrm{E}_{\mathrm{g}}, \mathrm{T}_{1 \mathrm{~g}},{ }^{1} \mathrm{~T}_{2 \mathrm{~g}}$ \\
\hline
\end{tabular}

Como a elaboração deste diagrama é extremamente trabalhosa, utilizamos normalmente o diagrama "pronto" de Tanabe-Sugano ${ }^{5}$, onde a unidade de energia é o parâmetro de repulsão intereletrônica do íon gasoso (parâmetro B), cujo valor varia para cada tipo de íon livre. O diagrama é apresentado de forma que a energia do estado fundamental é tomada como zero para todos os valores de campo, denominado de $\Delta$. Reproduzimos na Figura 2 o diagrama E/B vs $\Delta / \mathrm{B}$.

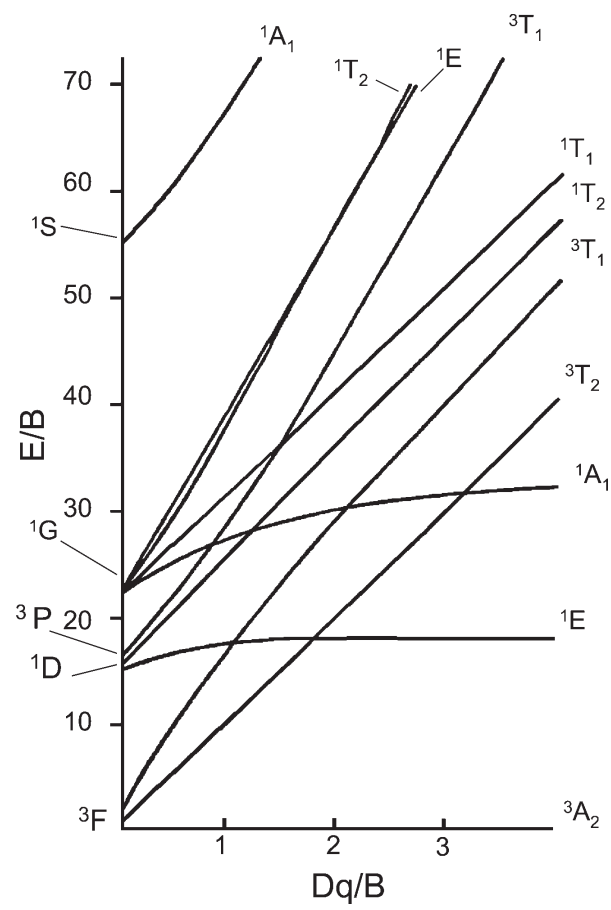

Figura 2. Diagrama de energia (Tanabe-Sugano) para íon $d^{8}$ em campo octaédrico

Pelo diagrama, observamos que no caso do complexo de $\mathrm{Ni}^{2+}$, o estado fundamental é o ${ }^{3} \mathrm{~A}_{2 \mathrm{~g}}$. As transições permitidas são aquelas que ocorrem entre o estado fundamental e os estados excitados que apresentam a mesma multiplicidade de spin. Em principio três transições são possíveis: ${ }^{3} \mathrm{~A}_{2 \mathrm{~g}} \rightarrow{ }^{3} \mathrm{~T}_{2 \mathrm{~g}},{ }^{3} \mathrm{~A}_{2 \mathrm{~g}} \rightarrow{ }^{3} \mathrm{~T}_{1 \mathrm{~g}}(\mathrm{~F})$ e ${ }^{3} \mathrm{~A}_{2 \mathrm{~g}} \rightarrow{ }^{3} \mathrm{~T}_{1 \mathrm{~g}}(\mathrm{P})$, onde $(\mathrm{F})$ e $(\mathrm{P})$ indicam os estados originados de ${ }^{3} \mathrm{~F}$ e ${ }^{3} \mathrm{P}$, respectivamente (ver a figura acima). Estas transições são também designadas pelas notações $v_{1}, v_{2}$ e $v_{3}$, respectivamente.

Dentre as transições esperadas, somente observamos $v_{3}$ e $v_{2}$. A transição $v_{1}$ ocorre numa região de energia em que os equipamentos convencionais, pelas suas limitações de natureza ótica, não podem detectar. Para contornar este problema podemos estimar, teoricamente, a posição desta banda $v_{1}$.

Os níveis de energia resultantes de todos os termos de RusselSaunders podem ser calculados ${ }^{6-8}$ e, no presente caso, aqueles de interesse são: 
${ }^{3} \mathrm{~A}_{2 \mathrm{~s}}-12 \mathrm{Dq}$

${ }^{3} \mathrm{~T}_{2 \mathrm{~g}}^{2 \mathrm{~g}}-2 \mathrm{Dq}$

${ }^{3} \mathrm{~T}_{1 \mathrm{~g}}$ (F) $7,5 \mathrm{~B}+3 \mathrm{Dq}+-1 / 2 \xi$

${ }^{3} \mathrm{~T}_{1 \mathrm{~g}}$ (P) $7,5 \mathrm{~B}+3 \mathrm{Dq}+1 / 2 \xi$

onde $\xi=\left(225 \mathrm{~B}^{2}+100 \mathrm{Dq}^{2}-180 \mathrm{DqB}\right)^{1 / 2}$. O parâmetro B acima mede a repulsão intereletrônica do complexo, também chamado de parâmetro de Racah, e o 10Dq é o parâmetro que dá o valor do desdobramento do campo cristalino ${ }^{6-8}$.

As energias de transição $v_{1}, v_{2}$ e $v_{3}$ são obtidas pela diferença de energia entre os níveis ${ }^{3} \mathrm{~T}_{2 \mathrm{~g}},{ }^{3} \mathrm{~T}_{1 \mathrm{~g}}(\mathrm{~F}) \mathrm{e}^{3} \mathrm{~T}_{1 \mathrm{~g}}(\mathrm{P})$ e o fundamental ${ }^{3} \mathrm{~A}_{2 \mathrm{~g}}$ e podem ser escritas da seguinte forma:

$$
\begin{array}{ll}
v_{1} & 10 \mathrm{Dq} \\
v_{2} & 7,5 \mathrm{~B}+15 \mathrm{Dq}-1 / 2 \xi \\
v_{3} & 7,5 \mathrm{~B}+15 \mathrm{Dq}+1 / 2 \xi
\end{array}
$$

Conhecendo-se a energia de transição $v_{1}$, os parâmetros Dq e B poderiam ser obtidos facilmente aplicando-se as equações acima. Entretanto, de maneira geral a transição $v_{1}$ não é observada na maioria dos espectrofotômetros disponíveis nos laboratórios que, como comentamos acima, não varrem a região do infravermelho próximo. A obtenção dos parâmetros acima, via cálculos, torna a tarefa extremamente trabalhosa. Entretanto, com o auxilio de programas aplicativos como Origin ${ }^{\circledR}$, muito conhecido e utilizado pelos estudantes, o trabalho pode ser bastante simplificado e rápido.

A título de exemplo, podemos demonstrar como isto pode ser feito. Dividindo-se $v_{3}$ por B e escrevendo a equação de forma explícita, temos:

$\frac{v_{3}}{\mathrm{~B}}=7,5+15 \frac{\mathrm{Dq}}{\mathrm{B}}+\frac{1}{2}\left(225-180 \frac{\mathrm{Dq}}{\mathrm{B}}+100\left(\frac{\mathrm{Dq}}{\mathrm{B}}\right)^{2}\right)^{1 / 2}$

Da mesma forma podemos, dividindo $v_{2}$ por B, escrever a expressão $\mathrm{v}_{2} / \mathrm{B}$ :

$\frac{v_{2}}{\mathrm{~B}}=7,5+15 \frac{\mathrm{Dq}}{\mathrm{B}}-\frac{1}{2}\left(225-180 \frac{\mathrm{Dq}}{\mathrm{B}}+100\left(\frac{\mathrm{Dq}}{\mathrm{B}}\right)^{2}\right)^{1 / 2}$

Como no presente caso os espectros fornecem os valores de $v_{3} \mathrm{e}$ $v_{2}$, uma forma conveniente para determinar os valores dos parâmetro Dq e B seria obter o gráfico $v_{3} / v_{2}$ versus Dq/B. "Chutamos" diversos valores da razão Dq/B e traçamos a curva mostrada na Figura 3.

Na curva obtida procuramos o valor da razão Dq/B que mais se aproxima do valor da razão $v_{3} / v_{2}$ experimental. Podemos então substituir o valor de Dq/B nas Equações 8 ou 9, e obter o valor de B. Substituindo-se o valor de B na equação abaixo (Equação 10), obtida pelo rearranjo das Equações 5, 6 e 7, podemos escrever:

$v_{2}+v_{3}-3 v_{1}=15 B$

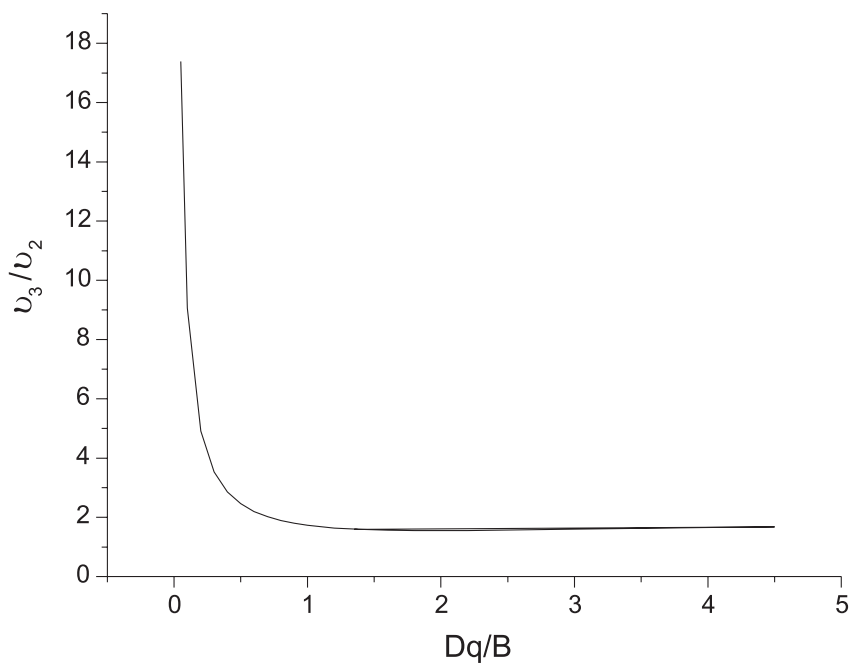

Figura 3. Variação da razão $v_{3} / v_{2}$ com $\mathrm{Dq} / \mathrm{B}$ para o complexo de $\mathrm{Ni}^{2+} \mathrm{em}$ campo octaédrico

Com estes procedimentos, todos os parâmetros foram determinados, desde que $v_{1}=10 \mathrm{Dq}$. Os valores calculados são apresentados na Tabelas $2 \mathrm{a}$ e $2 \mathrm{~b}$.

A Tabela $2 \mathrm{~b}$ nos mostra a intensidade de campo produzida pelos ligantes, baseada nos valores calculados de $v_{1}$ (10Dq). A seguinte ordem crescente de intensidade de campo produzida pelos ligantes é encontrada: en $>\mathrm{NH}_{3}>\mathrm{H}_{2} \mathrm{O}>$ dmso. A transição proibida por spin, ${ }^{3} \mathrm{~A}_{2 \mathrm{~g}} \rightarrow{ }^{1} \mathrm{E}_{\mathrm{g}}$ é também observada nos espectros, exceto no complexo de etilenodiamina. Esta transição foi atribuída baseada no diagrama de Tanabe-Sugano acima, com os valores de $\mathrm{Dq} / \mathrm{B}$ entre 0,85 a 1,4 .

A Tabela 2a lista os valores calculados de B, o parâmetro de repulsão intereletrônica. $\mathrm{O}$ valor deste parâmetro no íon gasoso, que mede a separação dos níveis ${ }^{3} \mathrm{~F}$ e ${ }^{3} \mathrm{P}$, é de $1080 \mathrm{~cm}^{-1}$. O fato do valor deste parâmetro ser menor nos complexos e com tendência em decrescer com o aumento da intensidade de campo produzida pelos ligantes reflete a natureza da ligação química. A diminuição do valor do parâmetro B nos complexos, em relação ao íon gasoso, pode ser explicada levando-se em consideração uma expansão da nuvem eletrônica dos orbitais d aumentando, assim, a distância média entre os elétrons. Esta expansão da nuvem eletrônica, chamada de "efeito

Tabela 2a. Valores calculados dos parâmetros de campo cristalino

\begin{tabular}{lcccc}
\hline & $v_{3} / v_{2}$ & $\mathrm{Dq} / \mathrm{B}$ & $\mathrm{v}_{3} / \mathrm{B}$ & $\mathrm{B} / \mathrm{cm}^{-1}$ \\
\hline$\left[\mathrm{Ni}\left(\mathrm{OH}_{2}\right)_{6}\right]^{2+}$ & 1,84 & 0,85 & 26,25 & 967 \\
{$\left[\mathrm{Ni}(\mathrm{dmsO})_{6}\right]^{2+}$} & 1,85 & 0,85 & 26,25 & 915 \\
{$\left[\mathrm{Ni}\left(\mathrm{NH}_{3}\right)_{6}\right]^{2+}$} & 1,60 & 1,35 & 34,15 & 820 \\
{$\left[\mathrm{Ni}(\mathrm{en})_{3}\right]^{2+}$} & 1,59 & 1,40 & 35,00 & 832
\end{tabular}

Tabela 2b. Valores calculados de $v_{1}$ e atribuições

\begin{tabular}{lcccc}
\hline & $v_{1} / \mathrm{cm}^{-1 *}$ & $v_{2} / \mathrm{cm}^{-1}$ & $v_{3} / \mathrm{cm}^{-1}$ \\
& ${ }^{3} \mathrm{~A}_{2 \mathrm{~g}} \rightarrow{ }^{3} \mathrm{~T}_{2 \mathrm{~g}}$ & ${ }^{3} \mathrm{~A}_{2 \mathrm{~g}} \rightarrow{ }^{1} \mathrm{E}_{\mathrm{g}}$ & ${ }^{3} \mathrm{~A}_{2 \mathrm{~g}} \rightarrow{ }^{3} \mathrm{~T}_{1 \mathrm{~g}}(\mathrm{~F})$ & 25381 \\
{$\left[\mathrm{Ni}(\mathrm{OH})_{6}\right]^{2+}$} & $8230(8500)^{\mathrm{a}}$ & 15037 & 13812 & 24038 \\
{$\left[\mathrm{Ni}(\mathrm{dmsO})_{6}\right]^{2+}$} & $7767(7728)^{\mathrm{b}}$ & 14286 & 12987 & 28011 \\
{$\left[\mathrm{Ni}\left(\mathrm{NH}_{3}\right)_{6}\right]^{2+}$} & $11053(10800)^{\mathrm{a}}$ & 12990 & 17452 & 29154 \\
{$\left[\mathrm{Ni}(\mathrm{en})_{3}\right]^{2+}$} & $11674(11500)^{\mathrm{a}}$ & - & 18349 & ${ }^{3} \mathrm{~A}_{1 \mathrm{~g}}(\mathrm{P})$ \\
\hline
\end{tabular}

*valor calculado; entre parentesis valores da literatura: ${ }^{a}$ ref. 9 , ${ }^{\text {bef. }} 10$ 
nefeulaxético" 10 deve-se ao grande espectroscopista Christian Klixbull Jorgensen (1931-2001), cujo perfil humano e científico foi recentemente publicado por Day ${ }^{11}$. Entretanto, devemos lembrar que no modelo original de campo cristalino idealizado por Bethe ${ }^{12}$ os átomos foram considerados como cargas pontuais, isto é, os mesmos se comportam como esferas rígidas indeformáveis.

\section{CONCLUSÕES}

O presente experimento, além de poder ser realizado em laboratório de graduação que disponha de um espectrofotômetro convencional, possui como objetivo central, introduzir o estudante ao modelo de campo cristalino, o qual permite interpretar razoavelmente os espectros eletrônicos de complexos de metais de transição. O experimento visa ainda motivar o estudante a compreender melhor a natureza das interações eletrônicas num sistema $\mathrm{d}^{\mathrm{n}}(\mathrm{n}>1)$ que dão origem aos diversos estados de Russel-Saunders, e de como eles são desdobrados pelos campos de diversas simetrias, produzidos pelos ligantes.

O presente experimento ilustra muito bem como podemos utilizar um modelo, idealizado inicialmente em 1929, e posteriormente modificado com introdução do parâmetro nefeulaxético (parâmetro de covalência), para correlacionar as medidas experimentais com a estrutura e natureza da interação num complexo metálico da primeira série de transição. Convém lembrar que experimentos envolvendo todos os complexos da primeira série de transição podem ser oferecidos para introduzir os fundamentos e técnicas da espectroscopia eletrônica.

\section{AGRADECIMENTOS}

$\mathrm{O}$ autor agradece à FAPESP e ao Pronex/CNPq pelo auxílio financeiro

\section{REFERÊNCIAS}

1. Atkins, P. W.: Jones, L; Chemistry, Molecules, Matter and Change, W.H. Freeman \& Co.: New York, 1997, cap. 21.

2. Shriver, D. F.; Atkins, P. W.; Langford, C. H.; Inorganic Chemistry, Oxford University Press, 1996, Parte 3.

3. Herzberg, G.; Atomic Spectra and Atomic Structure, Dover Publ.: New York, 1944, p. 128.

4. Cotton F. A.; Chemical Applications of Group Theory, John Willey \& Sons, $3^{\mathrm{a}}$ ed., 1990.

5. Tanabe, Y.; Sugano, S.; J. Phys. Soc. Jpn. 1954, 753, 766.

6. Jorgensen, C. K.; Adv. Chem. Phys. 1959, 2, 123.

7. Lever, A. B. P.; Inorganic Electronic Spectroscopy, Elsevier Sci. Publ. BV: Amsterdam, 1984.

8. Lever, A. B. P.; J. Chem. Educ. 1968, 45, 711.

9. Meek, D. W.; Drago, R. S.; Piper, T. S.; Inorg. Chem. 1962, 1, 285.

10. Jfrgensen, C. K.; Prog. Inorg. Chem. 1962, 4, 23.

11. Day, P.; Coord. Chem. Rev. 2003, 238-239, 3.

12. Bethe, H.; Ann. Physik 1929, 3, 133. 